\title{
Up Close and Personalized: advancing personalized medicine in the 21st century
}

The 4th Up Close and Personalized, International Congress on Personalized Medicine, Tel Aviv, Israel, 17-20 June 2015

The current article outlines the main issues presented during the 4th Up Close and Personalized (UPCP), International Congress on Personalized Medicine, which took place in Tel Aviv (Israel) on 17-20 June 2015. The main topics presented included: phenotypic and 'omics' data in the personalized medicine of diabetes, obesity and oncology; updated reports on large-scale human genetics applied to drug discovery and precision medicine; new advances in disease targeted nano delivery systems, lessons derived from big clinical data and barriers for implementing personalized medicine in at the point of care.

\section{Personalized medicine in metabolic diseases}

In an extraordinary plenary session, Philippe Froguel (Imperial College London, UK) brought new insight into the genetics of diabetes and obesity toward prediction and personalized medicine. Approximately 10\% of children and young diabetes cases have a monogenic cause, which can be elucidated, allowing a specific treatment. Accordingly, individuals diagnosed with $G C K$ gene mutation (MODY2/GCK) have no need for antidiabetic therapy. In contrast patients with other monogenic diabetes defects usually have severe insulin secretory defect and need treatment. Fortunately, most frequent MODY forms (MODY3/HNF1- $\alpha$, MODY12/ABCC8 and MODY13/KCNJ11) can be optimally treated by oral sulfonylurea drugs, while the other forms usually need insulin therapy. Froguel advises genetic testing for:

- Every child with diabetes before 6 months (1 year) of life;

- Every diabetic child/teen proven negative for INS or GAD auto-antibodies;

- Every individual with an early onset of Type 2 diabetes (T2D).
The reader is referred to useful website and app at [1].

Similarly, $5 \%$ of severe obesity cases are monogenic and prevalence increases to $30 \%$ in Pakistani consanguinous cases. Most frequent are mutations in LEP and MC4R; and genetic deficiency of leptin can be 'cured' by recombinant leptin therapy.

Froguel recommends that a monogenic form of obesity should be assessed:

- In every child with severe (hyperphagic) obesity before 3 years of life (especially when first obese in family) or with developmental delay;

- In severely obese individuals from consanguinous families.

However, genomics only modestly contribute to prediction of polygenic forms of T2D. The metabolomic profiling and epigenetics should be used on top of clinical and genetic risk factors to improve the early identification of at-risk subjects.

The power of epigenetics in the prediction, prevention and treatment of T2D was further discussed by Francesco Beguinot (University of Naples, Italy). His talk focused on $H O X$ genes at the interface between the unhealthy
Adriana Fodor ${ }^{*}, 1$, Naphtali Rishe $^{2}$ \& Eddy Karnieli ${ }^{3}$

'University of Medicine \& Pharmacy 'Iuliu Hatieganu', Cluj-Napoca, Romania ${ }^{2}$ Florida International University, School of Computing \& Information Sciences, Miami, FL, USA

${ }^{3}$ Galil Center for Telemedicine, Medical Informatics \& Personalized Medicine, Rappaport Faculty of Medicine, Technion, Haifa, Israel *Author for correspondence: adifodor@yahoo.com 
environment and the risk of obesity and T2D. In a mouse model of diet induced diabetes, high-fat diet induces HOXA5 promoter hypermethylation and lowtranscriptional activity in subcutaneous adipose tissue, while weight reduction diminished the methylation and rescued HOXA5 expression.

Eddy Karnieli (Technion, Haifa, Israel) gave a comprehensive overview on obesity pathophysiology, identifying several molecular targets for anti-obesity drugs. An interesting debate from the audience arouse at the end of his presentation, stressing the relative modest effect of existing anti-obesity drugs compared with bariatric surgery. Several possible explanations were discussed like the lack of personalization and predictive factors, role of microbiota and probably the need for blocking multiple pathways.

Hans Haring (University of Tübingen, Germany) focused on the question why lifestyle intervention does not fit all. Haring identified two subphenotypes of prediabetes, which respond differently to lifestyle intervention. Approximately $24 \%$ of diabetes prone population in southwest Germany, does not respond to lifestyle intervention. Phenotype of lifestyle nonresponders is characterized by:

- Fatty liver;

- Low 'physical fitness' (aerobic capacity);

- Exercise 'nonresponder' (do not increase muscle PGC1 $\alpha$ in response to exercise);

- Brain insulin resistance.

Derek LeRoith (Technion) discussed the role of dyslipidemia in carcinogenesis, emphasizing the need for an intensive and personalized statin therapy in oncologic patients, based on drug and tumor characteristics.

\section{Personalized medicine in cancer}

Salomon Stemmer (Tel Aviv University, Israel) addressed the major challenges of chemotherapy, such as, the delicate balance between benefit and risk, the lack of predictive biomarker, no target therapy and overall poor results. Much hope is awaited from immune therapy. Nivolumab/pembrolizumab are anti-PD-1 monoclonal antibodies that selectively block immune checkpoint pathways, restoring T-cell immune activity directed against the tumor cell. Two speakers (Juliane Stickel, Tübingen, Germany and Arie Admon, Technion) discussed the perspective of personalized cancer vaccine based on HLA peptidome or HLA ligandome analysis of the tumor cells. Both speakers agreed that vision of personalized cancer vaccine will soon become reality.

Yehuda Assaraf (Technion) addressed another challenge of current chemotherapy: the multidrug resistance. He proposed a model for hydrophobic weak base drug-induced lysosome-mediated multidrug resistance that can be overcome by lysosomal photo-destruction. Ronit Satchi-Fainaro (Tel Aviv University, Israel) showed in tumor dormancy models and in human tissue samples that miRNAs miR-34a, miR-93 and miR-200c are downregulated during osteosarcoma progression. Reconstitution of dormancy-associated miRNAs into cells has therapeutic potential by reverting cancer cells into a 'dormant-like' status. Avi Schroeder (Technion) highlighted the potential of nanocarrier-directed delivery system to target specific cancer cell with specific proteins or miRNAs.

\section{Large-scale human genetics applied to drug discovery \& personalized medicine}

Gad Rennert (Technion) outlined the main pitfalls of using large next-generation sequencing (NGS) in cancer: 1) processing individual list of variants is time consuming; 2) is complex and requires a major time investment of clinical and genomics professionals; 3) majority of mutations are rare and of unknown clinical significance.

Gabi Tarcic (NovellusDx, Israel) presented a novel precision cancer analysis system for identifying functional significance of mutations.

Alan R Shuldiner (Regeneron Genetics Center, NY, USA) followed by Andy Faucett, (Geisinger Health System, PA, USA) discussed the power of human genetics to accelerate target identification, validation and drug development. They presented unique collaboration between genetic center and healthcare system with longstanding electronic health records (EHR) and comprehensive clinical data. Such collaboration allows:

- Broad interrogation of clinical phenotypes for individuals with interesting genotypes;

- Understanding the contribution of genetic variation to longitudinal health outcomes, including medication-related outcomes (efficacy, adverse events).

Making genomic data medically actionable and translating genetic discoveries into patient care are going to improve the ability to predict and prevent disease and optimize treatments based on genetic information for each individual.

Scott DuVall (University of Utah, UT, USA) described a database of genetic and molecular diagnostic tests (GDx) linked to EHR of US Veterans Affairs (VA). He noted underutilization of cancer genetic testing within VA. Actually, only $26 \%$ of the patients 
diagnosed with breast cancer received $B R C A 1 / 2$ testing. He underscored the importance of developing decision support tools within the EHR.

Elliot Siegel (University of Maryland, MD, USA) discussed the challenges and achievement in applying big data analytic algorithms to computed tomography (CT) imaging for improved diagnosis.

\section{The benefits of big data}

Large healthcare systems with EHR accumulate huge amounts of longitudinal data that are merely underutilized. Making the effort to analyze big data may lead to development of algorithms/guidelines for screening, diagnosis and drug selection.

The use of big data was highlighted by Amanda Niskar (Arthritis Foundation, GA, USA), Yelena Yesha (University of Maryland, Baltimore County, MD USA) and Naphtali Rishe (Florida International University, FL, USA) for improving health outcomes for people with arthritis. According to them big data analytics hold promise to help us understand the many unanswered questions about the cause of the $>100$ types of arthritis and how to prevent, diagnose and treat arthritis. The VA Informatics and Computing Infrastructure (VINCI) national clinical database from the VA provides detailed patient information for approximately 35 million patients who received care at 152 medical centers and more than 800 outpatient clinics over the past 15 years.

One of the largest limiting factors in understanding rare disease is the scarcity of data.

Neta Zach (Prize4Life, Israel) described the PROACT Database of amyotrophic lateral sclerosis (ALS), with more than 8500 unique patient records available open access to researchers all around the world.

\section{Decision support healthcare systems}

Borko Furht (Florida Atlantic University, FL, USA) described Electronic Medical Assistant program which is an EHR system with a large library of built-in medical content designed to increase physician's efficiency and improve outcomes.

Varda Shalev (Maccabi Healthcare Services, Israel) presented an algorithm derived from epidemiological data (complete blood counts) that provides a personalized predictive risk score for colorectal cancer. Individuals above a predefined threshold are flagged in the

\section{Reference}

1 Genetic types of diabetes including maturity-onset diabetes of the young (MODY).

www.diabetesgenes.org
EHR system thus alerting their family practitioners to order colonoscopy.

A panel led by Nicki Liberman (Clalit Health System, Israel) and Eddy Karnieli discussed the barriers for implementing personalized medicine in primary practice reaching an important conclusion: the clinicians require informatics support and algorithms that work in background to assist in integrating all the clinical, molecular and 'omics' data toward a personalized care.

\section{US National Science Foundation industry- university centers}

Specials sessions and panels were conducted on collaboration opportunities between the US National Science Foundation (NSF) Industry/University Cooperative Research Centers and Israeli Academia and Industry. These sessions were attended by industrial board members and faculty of US NSF Industry/University Cooperative Research Center for Advanced Knowledge Enablement (I/UCRC-CAKE) and Center for Hybrid Multicore Productivity Research (I/UCRC-CHMPR) and a large group of Israeli academics and industrial $R \& D$ leaders. As an outcome of these sessions, further steps are being undertaken toward the establishment of NSF Centers' sites at the Technion and Tel Aviv University. US discussion facilitators included: CAKE Board: Tajana Lucic (NOA); CHMPR Board: Gabriel Silberman (Dell); CHMPR Faculty from University of Maryland and University of Utah: Yelena Yesha, Yacov Yesha, Eliot Siegel, Scott DuVall, Don Engel and Paul Mulhern; CAKE Faculty from Florida International and Florida Atlantic Universities: Naphtali Rishe and Borko Furht; and representatives from US foundations and companies. Israeli discussion facilitators were Eddy Karnieli (Technion) and Mira Marcus-Kalish (Tel Aviv University).

\section{Financial \& competing interests disclosure}

The author has no relevant affiliations or financial involvement with any organization or entity with a financial interest in or financial conflict with the subject matter or materials discussed in the manuscript. This includes employment, consultancies, honoraria, stock ownership or options, expert testimony, grants or patents received or pending, or royalties.

No writing assistance was utilized in the production of this manuscript. 